\title{
RELAÇõES ENTRE INTENSIDADE, DURAÇÃO E FREQUÊNCIA DAS PRECIPITAÇÕES MÁXIMAS DE 24 HORAS E EQUAÇÃO DE CHUVAS INTENSAS PARA A CIDADE DE UBERLÂNDIA-MG
}

\author{
PETRUCCI, Eduardo - eduardo11sp@hotmail.com \\ Universidade Federal de Uberlândia / UFU \\ OLIVEIRA, Luiz Antônio - luiz_ao@msn.com \\ Universidade Federal de Uberlândia / UFU
}

\begin{abstract}
RESUMO: O conhecimento sobre as precipitações máximas e estabelecimento de relações entre a intensidade, duração e frequência de ocorrência desses eventos são de suma importância no planejamento de sistemas de drenagem, na dinâmica hidrológica, no dimensionamento de obras de engenharia urbana, produção de energia e na irrigação. Diante da importância do tema tratado, o objetivo desse trabalho é estimar as relações entre a intensidade, duração e intercorrência da precipitação máxima a partir das curvas de IDF e calcular a Equação de Chuvas Intensas para a cidade de Uberlândia-MG. Como base de cálculo foi utilizada a série histórica de precipitação de 1981 a 2015, da estação convencional de Uberlândia-MG $\mathrm{n}^{\circ}$ 83527. Primeiramente foram determinadas as máximas anuais dos acumulados de chuvas de $24 \mathrm{~h}$, sendo posteriormente calculadas as intensidades horárias e em minutos. Na distribuição de probabilidades de chuvas e construção do IDF e da Equação de Chuvas Intensas para a cidade foi utilizada a função densidade de probabilidade de Gumbel. Determinadas as variáveis, a Equação de Chuvas Intensas construída é $\mathrm{I}=\left(330,4083 \times \mathrm{T}^{0,1452}\right) / \mathrm{t}^{0,6164}$, onde I é a intensidade da chuva, $\mathrm{T}$ é o período de retorno em anos e, $t$ é a duração em minutos. Pela análise do gráfico IDF, são esperadas chuvas mais intensas nos primeiros minutos de duração para períodos de retornos mais longos, principalmente a partir de 25 anos e, maiores volumes pluviométricos para elevados tempos de duração e retornos mais longos.
\end{abstract}

PALAVRAS-CHAVES: Precipitação; Chuvas Intensas; I-D-F; Uberlândia-MG.

THE RELATION BETWEEN INTENSITY, DURATION AND FREQUENCY OF 24 HOUR MAXIMUM PRECIPITATION AND RAINFALL EQUATION FOR UBERLÂNDIA-MG, PERIOD OF $1981-2015$

ABSTRACT: The knowledge concerning maximum rainfall and the relationship between the intensity, duration and frequency of the occurrence of these events are of paramount importance in the planning of drainage systems, hydrological dynamics, design of urban engineering works, energy production and in irrigation. Given the importance of the subject, the objective of this work is to estimate the relationships between the intensity, duration and occurrence of maximum precipitation from the IDF curves and to calculate the Heavy Rain Equation for the city of Uberlândia-MG. The basis of the calculation was based upon the historical series of precipitation from 1981 to 2015, from the conventional station of Uberlândia-MG $n \circ 83527$. Firstly, the annual maximum of $24 \mathrm{~h}$ accumulated rainfall was determined, and then the hourly intensities and minutes were calculated. In the distribution of rainfall probabilities and construction of the IDF and the Heavy Rain Equation for the city, the Gumbel probability density function was used. Given the variables, the constructed Heavy Rain Equation is $I=(330,4083 \quad x$ $\left.\mathrm{T}^{0,1452}\right) / \mathrm{t}^{0,6164}$, where $\mathrm{I}$ is the rainfall intensity, $\mathrm{T}$ is the return period in years, and $\mathrm{t}$ is the duration in minutes. By analyzing the IDF graph, more intense rainfall is expected in the first few minutes for longer return periods, especially from 25 years and higher rainfall for longer duration and longer returns.

KEYWORDS: Precipitation, Rainfall, I-D-F; Uberlândia-MG 


\section{INTRODUÇÃO}

O conhecimento sobre as variáveis climáticas, independente da escala de abordagem, constitui importante ferramenta aliada ao processo de planejamento das atividades que estruturam os espaços. A precipitação, uma das componentes do clima, exerce papel fundamental na organização das atividades humanas, na dinâmica hidrológica, agrícola, socioeconômica, urbana e muitos outros.

A quantidade de chuva precipitada não é homogênea no tempo e no espaço, podendo variar em função do total precipitado, da duração, intensidade e frequência de intercorrência desses eventos (HOLTZ, 1976; BERTONI E TUCCI, 2004; VAREJÃO-SILVA, 2006). O excesso ou a escassez de chuvas pode acarretar em desestabilização no sistema climático, com potencial de causar danos humanos e materiais, por meio dos desastres. De acordo com Santos e Galvani (2014), a precipitação é o atributo mais importante quando há o estudo climático de uma região.

A sapiência acerca das precipitações máximas e das chuvas intensas quanto à sua intensidade, duração do evento e a frequência de ocorrência desses eventos, são de suma importância em projetos envolvendo recursos hídricos, tais como: produção de energia, uso da água, produção agrícola e projetos de irrigação, dimensionamento de obras de engenharia, dentre outros, sendo que, eventos de chuvas intensas podem acarretar em desastres quando não houver um planejamento dessas obras que levem em consideração os volumes pluviométricos escoados na forma de lâminas de água. Com isso, obras de contenção, como piscinões, vertedouros ou barragens, bem como de canalização dos cursos d'água, bueiros, galerias de escoamento subsuperficial nos ambientes urbanos necessitam dos conhecimentos sobre os volumes pluviométricos, intensidade das precipitações intensas e a frequência com que esses eventos ocorrem. (MOHYMONT, DEMARÉE e FAKA, 2004; TUCCI, 2004; MORUZZI e OLIVEIRA, 2009; MACHADO e SILVA, 2011; OLIVEIRA et. al., 2011; SOUZA et. al., 2012; BACK, OLIVEIRA e HENN, 2012).

Os eventos extremos podem ser caracterizados por serem naturais ao sistema ambiental, indicando excepcionalidades e podem ser estimados por meio de análises estatísticas e estão relacionados com a variabilidade climática. De acordo com Liebmann, Jones e Carvalho (2001), conceituar o que é um evento extremo é uma tarefa complexa pois, deve-se levar em consideração as heterogeneidades dos espaços e dos fenômenos atmosféricos envolvidos. Por Evento Extremo, INPE (2009) considera como extremo os valores que se distanciam dos valores médios climáticos e podem ocorrer em diversas escalas temporais, sendo que, extremos a curto prazo estão relacionados com o tempo e os de médio prazo estão relacionados com o clima. Ambos são importantes e com poder de impactar as atividades humanas.

A Organização Meteorológica Mundial (OMM) definiu Normais Climatológicas (NC) - ou Normais de Referência - como "[...] médias de dados climatológicos computados por um período consecutivo de 30 anos" $^{1}$ (OMM, 1989 , p. 2). Com isso, são realizadas estatísticas de dados climatológicos, sobre uma determinada região por um período mínimo de 30 anos. Assim, quando ocorrer desvios positivos ou negativos em relação à média do período histórico

\footnotetext{
${ }^{1}$ Tradução dos autores.
} 
(NC), levando em consideração a magnitude os desvios, diz-se que ocorreram Eventos Climáticos Extremos (ECE) (INPE, 2009).

Santos e Galvani (2014) dissertam que "[...] os valores de precipitação que não se encontram dentro do intervalo da normal climatológica ou dos valores médios de uma determinada série histórica podem ser considerados como eventos extremos" (p. 215). Pruski et. al. (2006) disserta que, as chuvas intensas são aquelas cuja intensidade da chuva $(\mathrm{mm} / \mathrm{h})$ excede o valor de referência para a pluviometria da referida localidade. Considerando as NC e a variabilidade climática de uma determinada localidade, ocorrências de eventos caracterizados por elevada pluviosidade em um curto período com grande intensidade, são chamadas de chuvas intensas.

Entretanto, há o fator da heterogeneidade dos espaços. O que pode ser considerado um ECE e Chuva Intensa em uma região de baixa pluviosidade, pode ser considerado um evento normal em outra região de elevada pluviosidade. No intuito de padronizar a identificação e ocorrência das chuvas intensas, a Organização Meteorológica Mundial por meio do ETCCDI (Team on Climate Change Detection and Indices) considera ordenar a série de dados e estimar o limite de $95 \%$ superiores dos dias com os maiores volumes pluviométricos precipitados. Para chuvas extremamente intensas, é considerado - limiar de $99 \%$ dos dias com máximos de precipitação (TANK, ZWIERS e ZHANG, 2009, p. 51). De fato, tanto chuvas intensas quanto chuvas extremamente intensas podem acarretar impactos no ambiente a nas sociedades.

As consequências geradas pelos ECEs afetam diretamente na vida das pessoas, seja por eventos de chuvas intensas ou de seca. Quando provocam impactos generalizados, como perda de vidas humanas, danos materiais e imateriais, gerando mal-estar social, prejudicando 0 funcionamento da sociedade, diz-se que ocorreram Desastres Naturais, ou apenas Desastres (BRASIL, 2017, p. 29). É importante ressaltar que os desastres decorrentes de eventos extremos variam de acordo com a vulnerabilidade dos locais de ocorrência desses eventos.

As consequências das chuvas intensas, de acordo com o Anuário Brasileiro de Desastres Naturais de 2013 (BRASIL, 2014), são destacados as enxurradas, inundações, alagamentos, deslizamentos em massa e podem estar associados a outros tipos de eventos meteorológicos, como vendavais e tornados, por exemplo. Outrossim, a ocorrência, a permanência e a intensidade desse tipo de evento, pode acarretar em problemas de excedente hídrico, aumentando a umidade do solo e/ou vazões dos rios, comprometendo a produção agrícola além da manutenção da biodiversidade associada.

Ainda segundo o anuário, do total de desastres relacionados a chuvas intensas, 30,34\% ocorreram na Região Sudeste, totalizando 27 ocorrências apenas no ano de 2013. No Brasil, 16,39\% dos óbitos por desastres ocorreram em decorrência direta de chuvas intensas. Se considerar os eventos decorrentes de chuvas intensas, como enxurradas, inundações e deslizamentos, a porcentagem de óbitos se aproxima dos $80 \%$. Daí a importância de se realizar estudos relacionados aos eventos extremos de precipitação.

A partir da aplicação de métodos estatísticos em estudos de climatologia é possível traduzir/transformar dados brutos em informações climatológicas, de 
modo a embasar pesquisas que servem de base para o planejamento do ambiente, muito importantes no processo de tomada de decisão dos gestores e administradores públicos. (LAMBERT, COOPER e PAGH, 1998). Dessa forma, é importante que se tenha uma densa rede de estações de coleta de dados, que seja capaz de fornecer dados para qualquer bacia hidrográfica ou região de estudo. Além disso, a constante manutenção desses aparelhos e capacitação dos profissionais envolvidos, para que não sejam registrados dados errados ou que não haja falhas nos bancos de dados que possam comprometer a qualidade dos estudos.

A estatística é uma importante ferramenta aplicada na modelagem de dados climatológicos, possibilitando o entendimento do comportamento das variáveis climáticas, bem como a identificação de padrões e flutuações deste comportamento. Destarte, dados de precipitação podem ser utilizados para a determinação da Equação de Chuvas Intensas e produção dos gráficos de Intensidade, Duração e Frequência (IDF) das precipitações máximas, estimando o regime dos eventos extremos de chuva intensas, possibilitando o cálculo da frequência de ocorrência desses eventos ao longo do tempo e quais as magnitudes dos eventos de acordo com a duração (CAMPOS-ARANDA, 1978; KOUTSOYIANNIS, D.; KOZONIS, D.; MANETAS, 1998; MOHYMONT, DEMAR'EE e FAKA, 2004; BACK, OLIVEIRA e HENN, 2012).

Com isso, verifica-se a importância da manutenção de redes de estações de coleta e registro de dados, que mantêm alimentados e atualizados os bancos de dados para que sejam aplicados métodos estatísticos em estudos referentes aos extremos de chuvas, de modo a estimar com maior precisão as ocorrências desses fenômenos ao longo do tempo. Em posse desses estudos de probabilidade de ocorrências, podem ser tomadas medidas pró-ativas, no ato do planejamento pelos poderes públicos, de modo que sejam minimizados os desastres decorrentes de tais eventos.

Diante do exposto, o objetivo desse trabalho é estimar as relações entre a intensidade, duração e intercorrência da precipitação máxima a partir das curvas IDF e construir a Equação de Chuvas Intensas de Uberlândia-MG, utilizando-se de dados do período de 1981 a 2015.

\section{LOCALIZAÇÃO DA ÁREA DE ESTUDO E CARACTERÍSTICAS CLIMÁTICAS}

A área de estudo compreende a cidade de Uberlândia-MG que está inserida na mesorregião do Triângulo Mineiro/Alto Paranaíba. Está localizado entre as coordenadas $18^{\circ} 5^{\prime} 25^{\prime \prime} \mathrm{S}$ e $48^{\circ} 17^{\prime} 19^{\prime \prime} \mathrm{W}$, e altitude média de 865 metros.

De acordo com Instituto Brasileiro de Geografia e Estatística - IBGE (2018) a área municipal é de $4.115,206 \mathrm{~km}^{2}$ e com população estimada ao fim do ano de 2017 de 676.613 habitantes. Uberlândia é a maior e mais importante cidade do Triângulo Mineiro/Alto Paranaíba, concentrando cerca de $30 \%$ da população da mesorregião. 


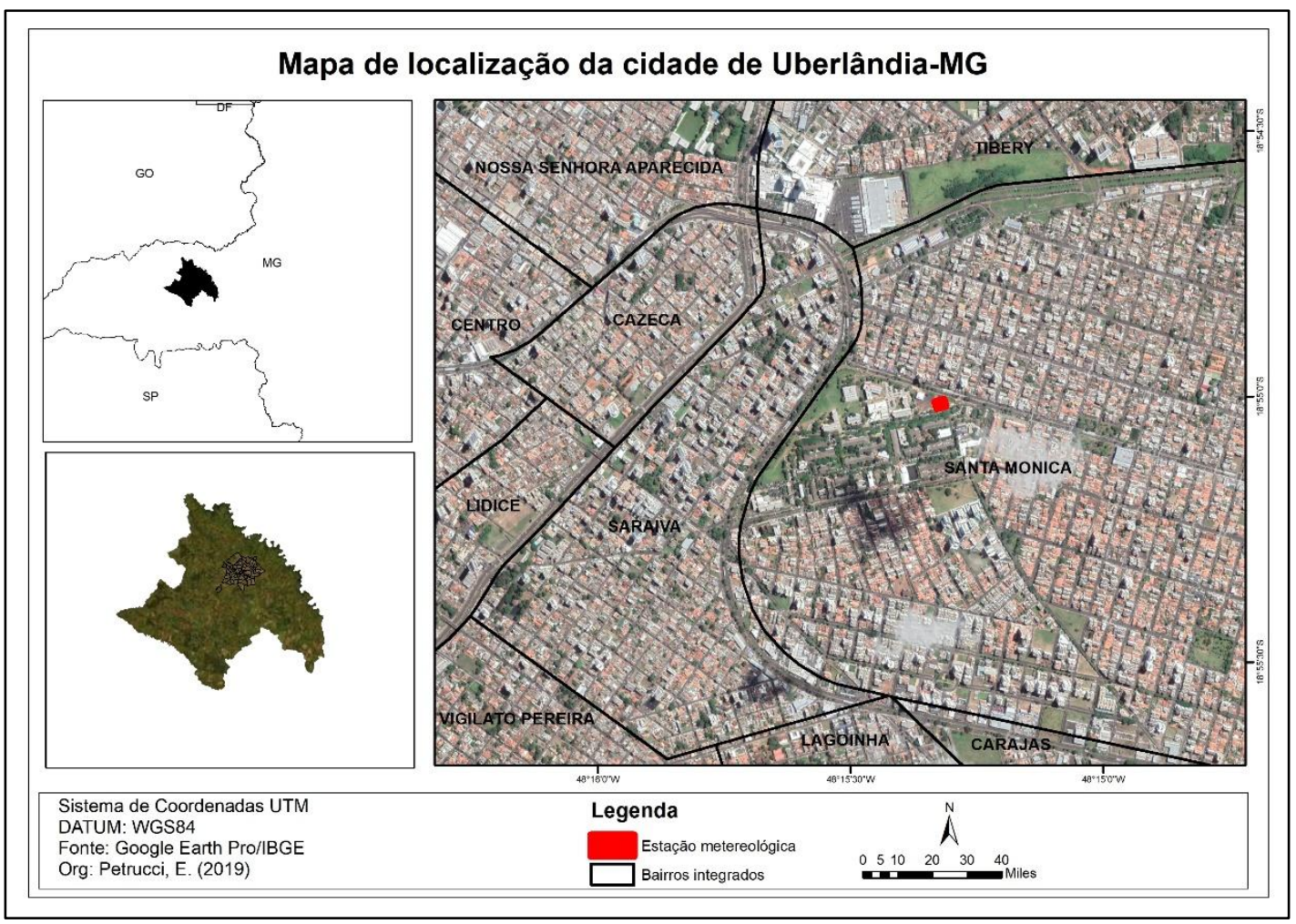

Figura 1 - Localização da cidade de Uberlândia-MG, com destaque para a localização da estação climatológica. Elaboração: PETRUCCI (2019)

De acordo com a classificação climática realizada por Köppen (1936), em que são levadas em consideração as variações sazonais e os valores médios mensais e anuais de temperatura e precipitação, a cidade de Uberlândia-MG está inserida numa zona Aw - megatérmico, tropical com verão chuvoso e inverno seco. Novais, Brito e Sanches (2018), ao proporem uma nova classificação climática para a mesorregião do Triângulo Mineiro/Alto Paranaíba, baseada na temperatura média do mês mais frio, variação na precipitação pluviométrica, quantidade de meses secos, passagens de frentes frias e possibilidade de formação de geadas, Uberlândia é classificada como Tropical Semiseco Meridional dos patamares do chapadão Uberlândia-Uberaba e do planalto de Araxá ( $\left.\mathrm{Tr}^{*} \mathrm{~m} 7\right)$, apresentando características de clima zonal quente, com 6 meses secos (abril a setembro), pluviosidade média anual próxima dos $1.600 \mathrm{~mm}$ e temperatura média do mês mais frio (agosto) acima de $18^{\circ} \mathrm{C}$. Na referida cidade, essas características são corroboradas pelo climograma de Uberlândia, pela figura 2 . 


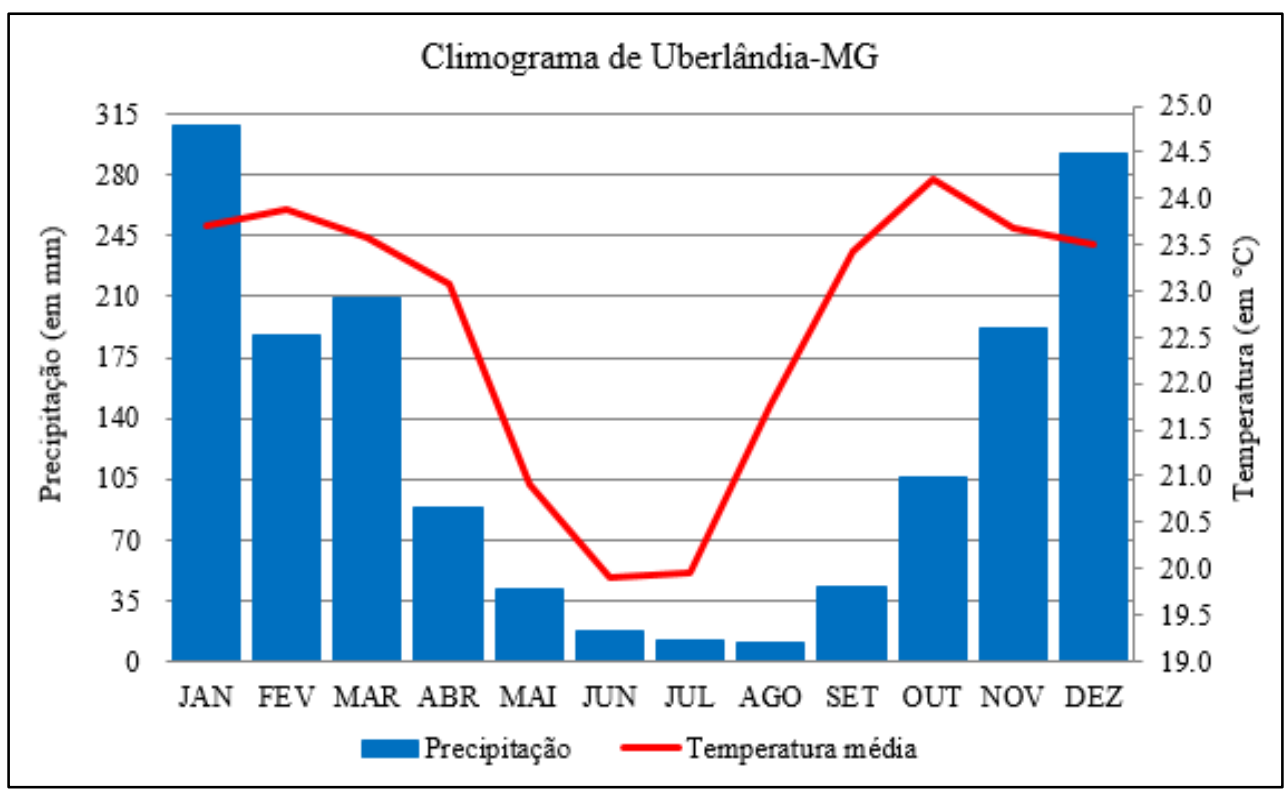

Figura 2 - Climograma da cidade de Uberlândia-MG, valores médios de temperatura média e precipitação de 1981 a 2015. Fonte: INMET (2017). Elaboração: PETRUCCI (2018)

Análise da figura 2 indica que as precipitações são concentradas nos meses de novembro a março, sendo que os maiores valores médios ocorrem nos meses de janeiro e dezembro, com 309 mm e 292 mm, respectivamente. No período de estiagem, que se estende dos meses abril a setembro, as médias dos totais de precipitações mantêm-se abaixo dos $100 \mathrm{~mm}$, sendo os meses de julho e agosto com os menores valores médios de precipitação, com $10 \mathrm{~mm}$ e $11 \mathrm{~mm}$, respectivamente. A precipitação média anual para a cidade de Uberlândia-MG é de $1.507 \mathrm{~mm}$.

Cavalcanti et. al. (2009) descreve sobre a transicionalidade climática característica da região Sudeste (incluindo também a cidade de Uberlândia-MG), limita-se entre os climas equatoriais e tropicais ao norte e subtropicais ao sul, sendo influenciada por sistemas atmosféricos oriundos de latitudes médias e tropicais. As chuvas são concentradas no verão, em detrimento de um inverno moderadamente frio e com baixa pluviosidade.

Concernente às temperaturas médias, os meses com maiores valores de temperatura compreendem o período de setembro a abril, com valores médios acima dos $23^{\circ} \mathrm{C}$, sendo os meses de outubro e fevereiro os com valores mais elevados, com $24,2{ }^{\circ} \mathrm{C}$ e $23,9^{\circ} \mathrm{C}$, respectivamente. No período de maio a agosto, as temperaturas são mais amenas, sendo os meses de junho e julho os mais frios, com $19,9^{\circ} \mathrm{C}$ e $20,0^{\circ} \mathrm{C}$, respectivamente. A temperatura média anual da cidade de Uberlândia-MG é de $22,6{ }^{\circ} \mathrm{C}$ e o gradiente entre o mês mais quente para o mês mais frio é de $4,3^{\circ} \mathrm{C}$. 


\section{MATERIAL E MÉTODOS}

Os dados de precipitação utilizados para construção dos gráficos IDF e respectiva equação de chuvas intensas foram coletados pela Estação Meteorológica de Observações de Superfície Convencional ( $n^{\circ}$ 83527) de Uberlândia-MG, localizada na Universidade Federal de Uberlândia (UFU), coordenadas $18^{\circ} 55^{\prime}$ de latitude $S$ e $48^{\circ} 17^{\prime}$ de longitude $W$, altitude de 872 metros.

Foram utilizados dados de precipitação máxima no intervalo de 24 horas, do período de 1981 a 2015. De uma série histórica de trinta e cinco anos de dados, ocorreram falhas nos meses de dezembro de 1984, setembro de 1985, maio e setembro de 1991, setembro de 1993 e abril de 2007 e, totalidade dos anos de 1986 a 1990, que correspondem a 13,4\% da amostra. Tucci (2004) aponta que falhas em dados de precipitação são permitidas desde que não ocorra em meses de maior volume pluviométrico da região pesquisada, ou seja, meses representativos, entretanto, é fundamental que as falhas sejam menor que $25 \%$ do total de dados para que não sejam geradas incertezas e calculados parâmetros que não representem a região de estudo.

A organização, tabulação dos dados e aplicação do método IDF foram feitas em planilha eletrônica do Microsoft Excel 2013. Foi utilizado o software IBM-SPSS Statistics 24.0 para encontrar as máximas alturas pluviométricas mensais e os meses de ocorrência, que serviram de base para a identificação do maior evento chuvoso no período de 24 horas de cada ano, ver tabela 2.

Concernente ao ajuste de dados de chuvas, Naghettini e Pinto (2007) apontam que as distribuições estatísticas de extremos utilizadas podem ser a distribuição Tipo I ou de Gumbel, a distribuição Log Normal, distribuição LogPearson III e Weibull. De acordo com Nerilo (1999) e Koutsoyiannis, Kozonis e Manetas (1998), a distribuição de Gumbel (ou Extrema Tipo 1) é a mais consagrada e é que mais se adequa para ajuste de dados, em que a função cumulativa de probabilidade dessa distribuição é dada pela equação 1.

$$
F_{(x)}=e^{-e^{-\left(\frac{x-\mu}{\alpha}\right)}}
$$

Onde:

$\mathrm{F}_{(\mathrm{x})}=$ é a função de densidade de probabilidade da distribuição de Gumbel;

$\mathrm{x}$ = é uma variável aleatória associada aos valores de precipitação máxima;

e $=$ é a base dos logaritmos neperianos.

$\mu$ e a = parâmetros da distribuição estimados com base na média e o desvio padrão dos valores da série de precipitações diárias máximas, respectivamente e, podem ser calculados pelas equações 2 e 3 :

$$
\alpha=\frac{\sqrt{6}}{\pi} * S
$$

Onde: $\pi(\mathrm{Pi})$ e, s é o desvio padrão; 


$$
\mu=\overline{\mathrm{x}}-0,5772 * \alpha
$$

Onde: $\bar{x}$ é a média dos valores da amostra da precipitação; $\alpha$ é a constante encontrada pela equação 2 .

Calculadas as variáveis das equações 2 e 3 bem como a média e o desvio padrão, foram obtidos os seguintes resultados: Média $(\overline{\mathbf{x}})=83,13 \mathrm{~mm}$; Desvio Padrão $(s)=21,97 ; \alpha=17,13 ; \mu=73,24$. Tendo sabidos os valores das variáveis, é possível determinar o cálculo das lâminas para distintas frequências e, com isso, encontrar a probabilidade de ocorrência de precipitação máxima para diferentes períodos de retorno $(2,5,10,25,50,75,100$ e 500 anos), ver tabela 3.

A variável reduzida (YT) utilizada para o cálculo do ajuste de dados pela distribuição de densidade de probabilidade de Gumbel, que varia em função do período de retorno (em anos) é dada pela equação 4:

$$
\mathrm{YT}=-\ln \left(\ln \left(\frac{\mathrm{y}}{(\mathrm{y}-1)}\right)\right)
$$

Onde: YT é o valor da variável reduzida, In é o logaritmo natural; y é o valor que corresponde ao período de retorno (em anos).

A variável $\mathrm{XT}^{\prime}$ (precipitação em $\mathrm{mm}$ de retorno prevista e que não será excedida) é determinada pela equação:

$$
\mathrm{XT}^{\prime}=\mu+(\alpha * \mathrm{YT})
$$

Onde: $\mathrm{XT}^{\prime}$ é o valor da precipitação máxima prevista; $\mu$ é o resultado da equação 3 ; $\alpha$ é o resultado da equação 2 ; YT é a variável reduzida encontrada pela equação 4.

Weiss (1964) ao estudar a precipitação anual de mais de mil estações de medição nos Estados Unidos, verificou que, ao adicionar $13 \%(* 1,13)$ ao valor da chuva anual corresponderá a magnitudes semelhantes do real. O valor desse incremento é muito próximo ao que é recomendado pela Companhia de Tecnologia de Saneamento Ambiental - CETESB (1986), que considera o incremento de $14 \%(* 1,14)$ nos valores de chuva significa valor mais próximo ao real. Com isso, após o cálculo das probabilidades de precipitação máxima para diferentes períodos de retorno, o valor de $X T^{\prime}$ (Precipitação em $\mathrm{mm}$ ) será multiplicado por 1,13, originando XT (precipitação em $\mathrm{mm}$ ) que corresponde ao valor de precipitação correspondente ao real.

Para determinar as concentrações de chuvas em intervalos de tempo horários, tendo-se apenas as concentrações diárias, adotou-se o método de desagregação de chuvas apresentado em Campos-Aranda (1978). O referido autor, após vários estudos visando avaliar o comportamento de precipitações, verificou que, durante o período de um dia (24 horas) a quantidade precipitada 
varia em função do tempo de ocorrência, sendo que esta é mais intensa em seus momentos iniciais. Assim, a desagregação representa a porcentagem de chuva precipitada à cada momento do evento, ver tabela 1 . Os coeficientes desagregados, constantes na tabela 1 , são bases para o cálculo da precipitação máxima com diferentes durações $(h)$ e respectivos períodos de retorno, tabela 4.

Tabela 1 - Relação entre a duração (horas) e coeficiente de precipitação

\begin{tabular}{ccccccccccc}
\hline \multicolumn{10}{c}{ Relação entre duração e coeficiente de precipitação } \\
\hline Horas & 1 & 2 & 3 & 4 & 5 & 6 & 8 & 12 & 18 & 24 \\
Coeficiente (\%) & 0,30 & 0,39 & 0,46 & 0,52 & 0,57 & 0,61 & 0,68 & 0,80 & 0,91 & 1,00 \\
\hline
\end{tabular}

Fonte: CAMPOS-ARANDA (1978)

No intuito de validação e calibração dos dados, mantendo relação entre a intensidade $(\mathrm{mm} / \mathrm{h})$ e a duração $(\mathrm{mim})$ em função do período de retorno, foi aplicado um Teste de Ajuste (Aderência) para cada período de retorno. Os produtos gerados da regressão são a Constante de Regressão $(A)$ e o Coeficiente de Regressão (B), parâmetros estes encontrados na equação da linha de tendência dos gráficos de regressão. O intuito de realizar regressão para cada período de retorno específico é que, no ato da substituição das variáveis pelos valores correspondentes, o parâmetro " $c$ " é resultado da média dos coeficientes de regressão (B) encontrados para cada retorno e não o coeficiente de regressão (B) encontrado quando realizada regressão geral para todos os períodos.

Após aplicar os testes de aderência para cada período de retorno $(2,5$, $10,25,50,75,100$ e 500 anos) e encontrar os parâmetros $A, B$ e $\operatorname{Ln}(A)$ específicos para cada retorno, foi calculada a intensidade com que essas precipitações ocorrerão para as diferentes durações (horas transformadas em minutos) para os retornos, ver tabela 5 . A intensidade pode ser calculada pela equação 8, com base nas representações matemáticas de Bernard (1932):

$$
\mathrm{I}=\frac{\mathrm{a} * \mathrm{~T}^{\mathrm{b}}}{\mathrm{t}^{\mathrm{c}}}
$$

Onde:I é a intensidade da chuva $(\mathrm{mm} / \mathrm{h})$; " $\mathrm{a}$ " é a constante de regressão; $\mathrm{T}^{\mathrm{b}}$ é o período de retorno elevado ao seu coeficiente de regressão; $t^{c}$ é a duração da chuva elevada ao seu coeficiente de regressão.

Por fim, foi aplicada uma regressão potência comum a todos as demais regressões potências dos períodos de retorno, ver tabela 7. A partir dessa aplicação é que foram conhecidos os parâmetros $A$, B e $\operatorname{Ln}(A)$ necessários para a composição da equação de intensidade de chuvas de Uberlândia-MG.

Montada a tabela de intensidade, é possível gerar o gráfico I-D-F (Intensidade-Duração-Frequência) das precipitações máximas. 


\section{RESULTADOS E DISCUSSÃO}

Os maiores volumes pluviométricos precipitados no período de 24 horas, em cada ano ao longo da série histórica, estão sumarizados na tabela 2.

Tabela 2 - Meses de ocorrência e a máxima altura pluviométrica ocorrida em cada ano

\begin{tabular}{cccccc}
\hline Ano & Mês & $\begin{array}{c}\text { Precipitação } \\
(\mathrm{mm})\end{array}$ & Ano & Mês & $\begin{array}{c}\text { Precipitação } \\
(\mathrm{mm})\end{array}$ \\
\hline 1981 & Dez & 79,4 & 2001 & Dez & 63,2 \\
1982 & Jan & 94,4 & 2002 & Jan & 147,0 \\
1983 & Jan & 126,8 & 2003 & Nov & 92,6 \\
1984 & Jan & 77,2 & 2004 & Dez & 99,4 \\
1985 & Jan & 74,4 & 2005 & Jan & 54,8 \\
1991 & Abr & 69,0 & 2006 & Dez & 83,9 \\
1992 & Fev & 83,6 & 2007 & Dez & 115,8 \\
1993 & Fev & 66,4 & 2008 & Dez & 81,0 \\
1994 & Mar & 76,4 & 2009 & Nov & 74,0 \\
1995 & Fev & 69,0 & 2010 & Nov & 71,8 \\
1996 & Dez & 48,0 & 2011 & Abr & 56,8 \\
1997 & Dez & 100,7 & 2012 & Dez & 89,2 \\
1998 & Out & 68,1 & 2013 & Mai/Out & 82,0 \\
1999 & Nov & 114,6 & 2014 & Nov & 72,3 \\
2000 & Dez & 98,2 & 2015 & Jan & 64,0 \\
\hline
\end{tabular}

Fonte: PETRUCCI (2018)

Com base nos dados apresentados na tabela 2, percebe-se uma considerável variação interanual entre as precipitações máximas. As máximas anuais variaram entre 48,0 e 147,0 mm, ocorridas em dez/1996 e jan/2002, respectivamente. As maiores concentrações em 24 horas foram de 147,0 e $126,8 \mathrm{~mm}$, ocorridas respectivamente nos meses de janeiro de 2002 e 1983 . O mês de dezembro é o mais recorrente.

Destaque para o ano de 2013, em que a precipitação máxima de 82,0 $\mathrm{mm}$ ocorreu duas vezes em dias distintos, sendo uma ocorrência no mês de maio e a outra no mês de outubro. Observa-se ainda o trimestre novembrodezembro-janeiro concentrou os maiores valores, devido a isso, deve-se atentar, sobretudo, pelo potencial gerador de desastres, tais como deslizamentos, enchentes, inundações, causando perdas materiais e danos ambientais. Esses valores devem ser considerados quando no ato da gestão urbana, elaboração de obras públicas e de infraestrutura, seja para contenção/minimização de problemas decorrente desses eventos.

Após a identificação das ocorrências de precipitações máximas em 24 horas para todos os anos da série histórica, foi aplicada a distribuição dos Extremos tipo 1, ou Gumbel para encontrar as probabilidades de ocorrência da precipitação máxima para distintos períodos de retorno (anos). Na tabela 3 estão sumarizados os valores das variáveis reduzidas, calculadas pela equação 4 , as probabilidades de ocorrência e os incrementos de $13 \%$ nos valores de precipitação. 
Tabela 3 - Cálculo das lâminas de precipitação máxima e respectiva probabilidade para os distintos períodos de retorno

\begin{tabular}{ccccc}
\hline $\begin{array}{c}\text { Período } \\
\text { de } \\
\text { Retorno }\end{array}$ & $\begin{array}{c}\text { Variável } \\
\text { reduzida }\end{array}$ & $\begin{array}{c}\text { Precipitação } \\
(\mathrm{mm})\end{array}$ & $\begin{array}{c}\text { Probabilidade } \\
(\text { Gumbel })\end{array}$ & $\begin{array}{c}\text { Correção } \\
\text { intervalo } \\
(* 1.13)\end{array}$ \\
\hline Anos & $\mathrm{YT}$ & $\mathrm{XT}^{\prime}(\mathrm{mm})$ & $\mathrm{F}(\mathrm{XT})$ & $\mathrm{XT}(\mathrm{mm})$ \\
2 & 0,3665 & 79,5218 & 0,5000 & 89,8597 \\
5 & 1,4999 & 98,9415 & 0,8000 & 111,8039 \\
10 & 2,2504 & 111,7991 & 0,9000 & 126,3329 \\
25 & 3,1985 & 128,0446 & 0,9600 & 144,6904 \\
50 & 3,9019 & 140,0965 & 0,9800 & 158,3090 \\
75 & 4,3108 & 147,1015 & 0,9867 & 166,2246 \\
100 & 4,6001 & 152,0593 & 0,9900 & 171,8270 \\
500 & 6,2136 & 179,7037 & 0,9980 & 203,0652 \\
\hline
\end{tabular}

Fonte: PETRUCCI (2018)

Utilizando os coeficientes da desagregação de chuvas propostos por Campos-Aranda (1978), apresentados na tabela 1, é estimada a precipitação máxima $(\mathrm{mm})$ para os distintos períodos de retorno (anos) em função da duração (h). Ver tabela 4.

Tabela 4 - Precipitação Máxima $(\mathrm{mm})$ para diferentes períodos de retorno em função do tempo de duração (horas)

\begin{tabular}{cccccccccc}
\hline \multirow{2}{*}{$\begin{array}{c}\text { Duração Coeficiente } \\
(\mathrm{h})\end{array}$} & \multicolumn{8}{c}{ Período de Retorno (Anos) } \\
\cline { 3 - 10 } & 2 & 5 & 10 & 25 & 50 & 75 & 100 & 500 \\
\hline 24 & $100 \%$ & 89,9 & 111,8 & 126,3 & 144,7 & 158,3 & 166,2 & 171,8 & 203,1 \\
18 & $91 \%$ & 81,8 & 101,7 & 115,0 & 131,7 & 144,1 & 151,3 & 156,4 & 184,8 \\
12 & $80 \%$ & 71,9 & 89,4 & 101,1 & 115,8 & 126,7 & 133,0 & 137,5 & 162,5 \\
8 & $68 \%$ & 61,1 & 76,0 & 85,9 & 98,4 & 107,7 & 113,0 & 116,8 & 138,1 \\
6 & $61 \%$ & 54,8 & 68,2 & 77,1 & 88,3 & 96,57 & 101,4 & 104,8 & 123,9 \\
5 & $57 \%$ & 51,2 & 63,7 & 72,0 & 82,5 & 90,2 & 94,8 & 98,0 & 115,8 \\
4 & $52 \%$ & 46,7 & 58,1 & 65,7 & 75,2 & 82,3 & 86,4 & 89,4 & 105,6 \\
3 & $46 \%$ & 41,3 & 51,4 & 58,1 & 66,6 & 72,8 & 76,5 & 79,0 & 93,4 \\
2 & $39 \%$ & 35,1 & 43,6 & 49,3 & 56,4 & 61,7 & 64,8 & 67,0 & 79,2 \\
1 & $30 \%$ & 27,0 & 33,5 & 37,9 & 43,4 & 47,5 & 49,9 & 51,6 & 61,0 \\
\hline
\end{tabular}

Fonte: PETRUCCI (2018)

Pela tabela 4, é notória a relação proporcional entre o aumento dos máximos de precipitação prevista com o avanço dos períodos de retorno, de forma mais significativa a partir dos 25 anos de retorno. A mesma porcentagem de aumento de quantidade de precipitação, entre o mesmo intervalo de duração (horas) são os mesmos para os períodos de retorno de 2, 25, 75 e 100 anos. Com isso, pode-se dizer que, os volumes pluviométricos estimados para 0 retorno de 25 anos, são $61 \%$ maiores em relação ao retorno de 2 anos, para o mesmo intervalo de duração. 14,88\% maiores para o retorno de 75 anos em relação ao retorno de 25 anos e, 3,38\% maiores para o retorno de 100 anos em comparação com o retorno de 75 anos.

Com os valores das precipitações esperadas em função da duração (h), é possível calcular as intensidades com que essas chuvas ocorrerão em determinada duração para os períodos de retorno. Ver tabela 5 . 
Tabela 5 - Intensidade de chuva $(\mathrm{mm} / \mathrm{h})$ segundo a Duração $(\mathrm{h})$ e Período de Retorno (anos)

\begin{tabular}{cccccccccc}
\hline \multicolumn{3}{c}{$\begin{array}{c}\text { Tempos de } \\
\text { duração }\end{array}$} & \multicolumn{10}{c}{ Período de Retorno (Anos) } \\
\hline Horas & Minutos & 2 & 5 & 10 & 25 & 50 & 75 & 100 & 500 \\
\hline 24 & 1440 & 3,74 & 4,66 & 5,26 & 6,03 & 6,60 & 6,93 & 7,16 & 8,46 \\
18 & 1080 & 4,54 & 5,65 & 6,39 & 7,31 & 8,00 & 8,40 & 8,69 & 10,27 \\
12 & 720 & 5,99 & 7,45 & 8,42 & 9,65 & 10,55 & 11,08 & 11,46 & 13,54 \\
8 & 480 & 7,64 & 9,50 & 10,74 & 12,30 & 13,46 & 14,13 & 14,61 & 17,26 \\
6 & 360 & 9,14 & 11,37 & 12,84 & 14,71 & 16,09 & 16,90 & 17,47 & 20,64 \\
5 & 300 & 10,24 & 12,75 & 14,40 & 16,49 & 18,05 & 18,95 & 19,59 & 23,15 \\
4 & 240 & 11,68 & 14,53 & 16,42 & 18,81 & 20,58 & 21,61 & 22,34 & 26,40 \\
3 & 180 & 13,78 & 17,14 & 19,37 & 22,19 & 24,27 & 25,49 & 26,35 & 31,14 \\
2 & 120 & 17,52 & 21,80 & 24,63 & 28,21 & 30,87 & 32,41 & 33,51 & 39,60 \\
1 & 60 & 26,96 & 33,54 & 37,90 & 43,41 & 47,49 & 49,87 & 51,55 & 60,92 \\
\hline
\end{tabular}

Fonte: PETRUCCI (2018)

Como os dados constantes na tabela 5 são derivados daqueles de precipitação máxima, (tabela 4), os comportamentos das variáveis em função do tempo de ocorrência e do período de retorno apresentam relação. As maiores intensidades são esperadas nas 3 primeiras horas de duração dos eventos de chuvas intensas, com diminuição gradual a partir dessa duração, entretanto, a diferença entre as intensidades é maior entre as durações entre 1 e 2 horas, para todos os períodos de retorno, sendo, para períodos de retorno mais longos. Inversamente, o volume pluviométrico esperado é maior nas horas finais do período de 24 horas, que corresponde a $100 \%$ do volume a ser precipitado, e são estimados menores valores de intensidades.

Após essa etapa, foi aplicado testes de aderência. A tabela 6 agrupa os coeficientes de determinação das retas de regressão: Exponencial, Linear, Polinomial e Potência, sendo que, quanto mais próximo de 1 , melhor é o ajuste de dados.

Tabela 6 - Coeficientes de determinação $\left(r^{2}\right)$ para diferentes testes de aderência

\begin{tabular}{ccccc}
\hline Exponencial & Linear & Logarítmica & Polinomial & Potência \\
0,8479 & 0,5856 & 0,9155 & 0,8281 & 0,9994 \\
\hline
\end{tabular}

Fonte: PETRUCCI (2018)

Pela tabela 6, verifica-se que o melhor ajuste se deu com a função Potência, sendo que, as funções Exponencial, Logarítmica e Polinomial também apresentaram elevados valores de $r^{2}$. Apenas a função Linear não se ajustou bem aos dados.

A intenção da aplicação desses testes foi, além de validação dos dados, encontrar os valores das variáveis $A, B$ e $\operatorname{Ln}(A)$, que foram base para a construção da equação de chuvas intensas. Após encontrar na função Potência a melhor aderência, foram aplicadas regressões em função de cada retorno, realizou-se uma regressão geral para todos os períodos de retorno. Na tabela 7 é apresentada a regressão tipo potência geral e os valores de $A, B$ e $\operatorname{Ln}(A)$ encontrados. Na figura 3 as linhas de tendência entre os dados observados em comparação com os dados modelados. 
Tabela 7 - Regressão (potência) para todos os períodos de retorno

\begin{tabular}{|c|c|c|c|c|c|c|}
\hline \multicolumn{7}{|c|}{ Regressão potencial } \\
\hline No & $x$ & $y$ & $\operatorname{Ln}(x)$ & $\operatorname{Ln}(y)$ & $\operatorname{Ln}(x) * \operatorname{Ln}(y)$ & $(\operatorname{Ln}(x))^{2}$ \\
\hline 1 & 2 & 339,7168 & 0,6931 & 5,8281 & 4,0397 & 0,4805 \\
\hline 2 & 5 & 422,6776 & 1,6094 & 6,0466 & 9,7316 & 2,5903 \\
\hline 3 & 10 & 477,6049 & 2,3026 & 6,1688 & 14,2041 & 5,3019 \\
\hline 4 & 25 & 547,0057 & 3,2189 & 6,3045 & 20,2933 & 10,3612 \\
\hline 5 & 50 & 598,4911 & 3,9120 & 6,3944 & 25,0151 & 15,3039 \\
\hline 6 & 75 & 628,4165 & 4,3175 & 6,4432 & 27,8185 & 18,6407 \\
\hline 7 & 100 & 649,5964 & 4,6052 & 6,4764 & 29,8247 & 21,2076 \\
\hline 8 & 500 & 767,6930 & 6,2146 & 6,6434 & 41,2861 & 38,6214 \\
\hline \multirow[t]{2}{*}{ Soma } & 767 & 4431,2020 & 26,8733 & 50,3053 & 172,2131 & 112,5074 \\
\hline & $\operatorname{Ln}(A)=$ & 5,8003 & $A=$ & 330,4083 & $B=$ & 0,1452 \\
\hline
\end{tabular}

Fonte: PETRUCCI (2017)

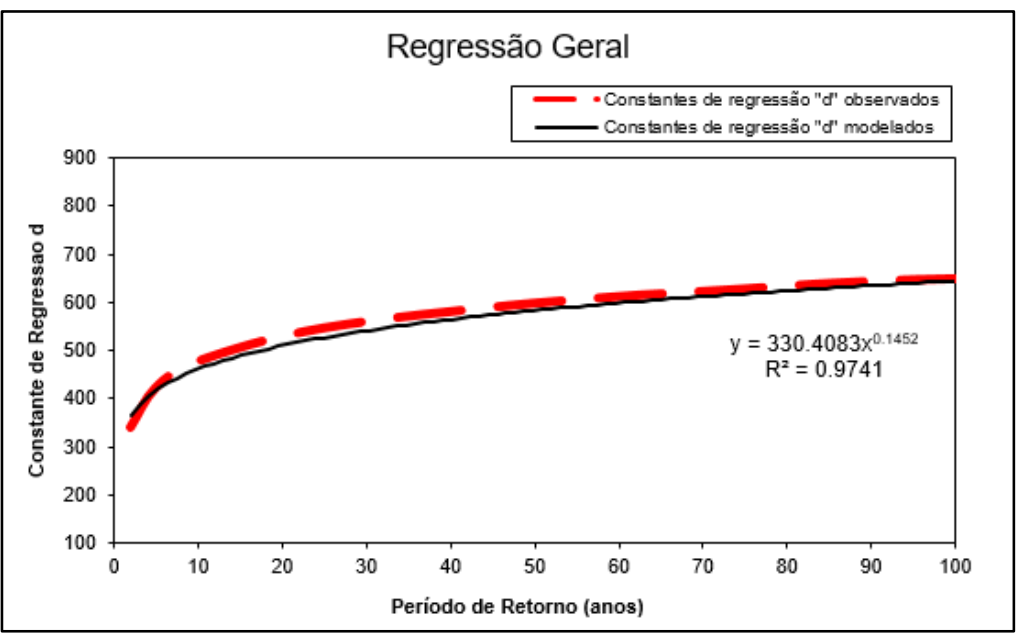

Figura 3 - Intensidade estimada para todos os períodos de retorno, acumulado de 24 horas. Fonte: PETRUCCI (2018)

Pela tabela 7, verifica-se os valores de A: 330,4083, B: 0,1452, e $\operatorname{Ln}(A)$ : 5,8003 . O valor de "c", necessário para completar a equação 6 é obtido pela média dos coeficientes de regressão $B$ obtidos para as regressões potência de todos os períodos de retorno separadamente, que foi de $-0,6164$. Sabidos os valores das variáveis, substitui-se na equação 6 , resultando na Equação de Chuvas Intensas para a cidade de Uberlândia-MG.

$$
I=\frac{330,4083 * T^{0,1452}}{t^{0,6164}}
$$

Onde: "I" é a intensidade da chuva $(\mathrm{mm} / \mathrm{h})$; "330,4083" é a constante de regressão para todos os períodos de retorno; "T" é o período de retorno (anos); " 0,1452 " é o coeficiente de regressão de todos os períodos de retorno; " $t$ " é tempo de duração da precipitação (minutos); "0,6164" é o coeficiente de regressão aplicado à cada período de retorno. 
Inserindo os valores de " $T$ " e " $t$ " na equação de chuvas intensas é possível calcular os distintos tempos de duração, bem como seus períodos de retorno, cujos resultados estão na tabela 8, e sua representação na figura 4.

Tabela 8 - Tabela de I-D-F para as chuvas intensas de Uberlândia-MG

\begin{tabular}{|c|c|c|c|c|c|c|}
\hline \multicolumn{7}{|c|}{ Intensidade - Duração - Frequência (IDF) } \\
\hline \multirow{2}{*}{$\begin{array}{l}\text { Frequência } \\
\text { (anos) }\end{array}$} & \multicolumn{6}{|c|}{ Duração em minutos } \\
\hline & 5 & 10 & 15 & 20 & 25 & 30 \\
\hline 2 & 135,4979 & 88,3857 & 68,8402 & 57,6542 & 50,2455 & 44,9046 \\
\hline 5 & 154,7831 & 100,9655 & 78,6381 & 65,8601 & 57,3969 & 51,2958 \\
\hline 10 & 171,1752 & 111,6581 & 86,9661 & 72,8349 & 63,4754 & 56,7282 \\
\hline 25 & 195,5383 & 127,5502 & 99,3438 & 83,2014 & 72,5097 & 64,8023 \\
\hline 50 & 216,2464 & 141,0582 & 109,8647 & 92,0127 & 80,1888 & 71,6650 \\
\hline 75 & 229,3622 & 149,6136 & 116,5282 & 97,5934 & 85,0524 & 76,0117 \\
\hline 100 & 239,1477 & 155,9967 & 121,4997 & 101,7571 & 88,6810 & 79,2546 \\
\hline 500 & 302,1164 & 197,0714 & 153,4912 & 128,5502 & 112,0312 & 100,1227 \\
\hline \multicolumn{7}{|c|}{ Intensidade - Duração - Frequência (IDF) } \\
\hline \multirow{2}{*}{$\begin{array}{l}\text { Frequência } \\
\text { (anos) }\end{array}$} & \multicolumn{6}{|c|}{ Duração em minutos } \\
\hline & 35 & 40 & 45 & 50 & 55 & 60 \\
\hline 2 & 40,8344 & 37,6080 & 34,9745 & 32,7753 & 30,9053 & 29,2914 \\
\hline 5 & 46,6463 & 42,9607 & 39,9523 & 37,4402 & 35,3040 & 33,4604 \\
\hline 10 & 51,5863 & 47,5104 & 44,1834 & 41,4052 & 39,0428 & 37,0040 \\
\hline 25 & 58,9285 & 54,2725 & 50,4719 & 47,2983 & 44,5997 & 42,2707 \\
\hline 50 & 65,1692 & 60,0201 & 55,8171 & 52,3074 & 49,3229 & 46,7473 \\
\hline 75 & 69,1218 & 63,6605 & 59,2025 & 55,4799 & 52,3145 & 49,5826 \\
\hline 100 & 72,0708 & 66,3765 & 61,7283 & 57,8469 & 54,5464 & 51,6980 \\
\hline 500 & 91,0474 & 83,8537 & 77,9817 & 73,0783 & 68,9087 & 65,3103 \\
\hline
\end{tabular}

Fonte: PETRUCCI (2018)

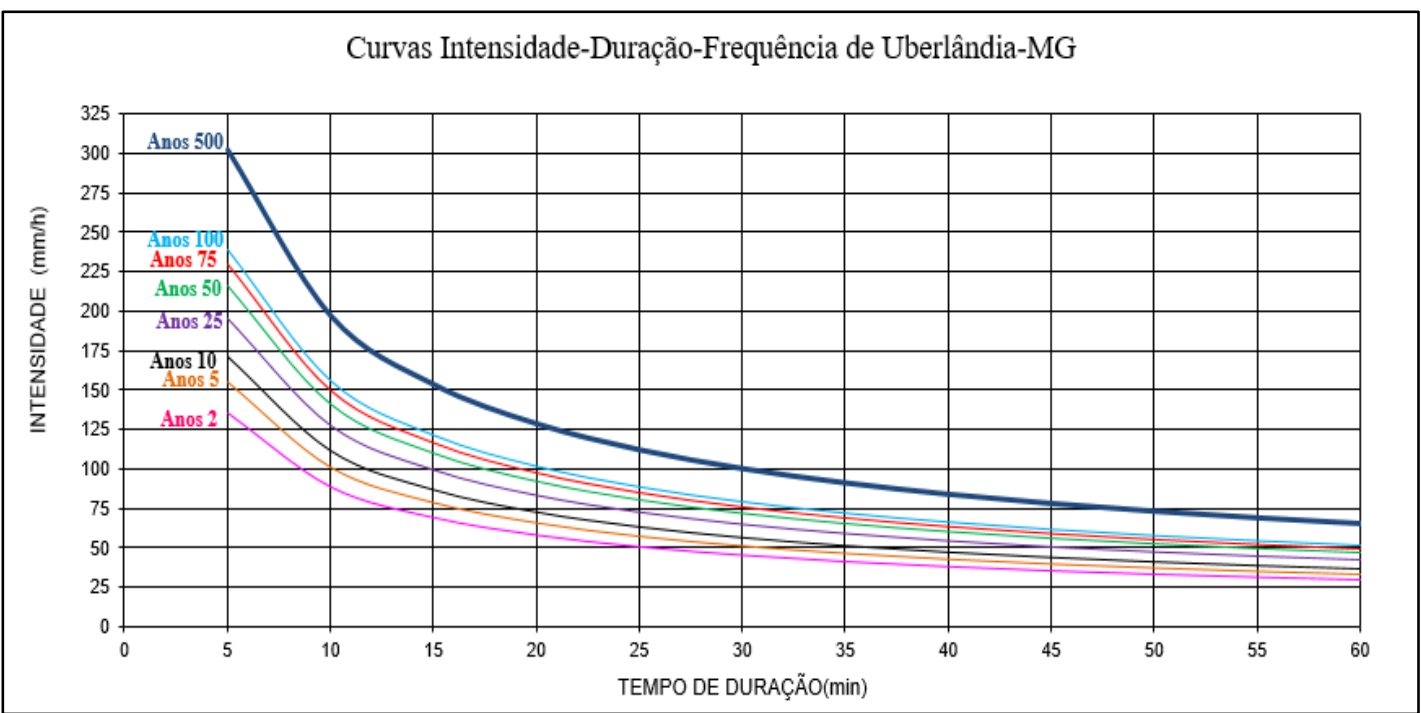

Figura 4 - Curvas I-D-F das Chuvas Intensas de Uberlândia-MG. Fonte: PETRUCCI (2018)

As intensidades apresentam relação direta com o aumento do período de retorno e, inversa em função do aumento da duração. Com isso, são esperadas 
chuvas mais intensas nos primeiros minutos de sua ocorrência e em períodos de retorno mais longos. Por exemplo, a intensidade verificada para o período de retorno de 10 anos, nos 5 primeiros minutos é de $171 \mathrm{~mm} / \mathrm{h}$, passando para 56 $\mathrm{mm} / \mathrm{h}$ nos $30 \mathrm{mim}$ e, para a duração de 60 minutos (1 hora) a intensidade diminui para $37 \mathrm{~mm} / \mathrm{h}$. A partir disso, o volume pluviométrico para o retorno de 10 anos, diminui cerca de $80 \%$ no limite da primeira hora de duração.

Pela figura 4, ao longo dos períodos de retorno são esperadas intensidade cada vez maiores, por exemplo, para o retorno de 500 anos, são esperados precipitação de $300 \mathrm{~mm}$, com forte concentração nos 10 primeiros minutos de duração. Para os períodos de 50, 75 e 100 anos, as estimativas são parecidas.

De modo geral, para todos os períodos de retorno as intensidades são mais fortes nos 10 primeiros minutos, sendo que, para a frequência de 2 anos a curva de IDF é mais suavizada do que para a frequência de 500 anos, que apresentou forte diminuição da intensidade a partir dos 20 primeiros minutos da precipitação e, a curva de IDF após os 20 minutos são mais suavizadas.

\section{CONSIDERAÇÕES FINAIS}

A metodologia empregada foi satisfatória e permitiu gerar a Equação de Chuvas Intensas e estimar graficamente as relações entre a intensidade, duração e frequência (IDF) das precipitações máximas do período de 24 horas para a cidade de Uberlândia-MG, em dados do período de 1981 a 2015.

Os resultados obtidos sobre as intensidades com que os eventos de precipitação máxima, variando em função das durações e dos períodos de retorno, são importantes e auxiliam no dimensionamento de obras de engenharia urbana, de modo que, quando levadas em consideração no ato do planejamento pelos gestores e administradores urbanos, promovem a tomada de medidas preventivas ao invés de medidas corretivas. Assim, diminuindo os gastos públicos com medidas corretivas provocadas pelos desastres decorrentes das chuvas intensas.

Concernente às consequências desses ECEs, além dos desastres gerados em obras hidráulicas, devem ser levadas em consideração também as contaminações por doenças da população que entra em contato com as enchentes, enxurradas e os alagamentos, acarretando em maiores gastos com saúde pública. Além das doenças relacionadas, as lâminas de água escoadas principalmente nas primeiras horas de duração dos eventos de chuva intensas causam danos materiais, principalmente nas áreas centrais das cidades.

Ressalta-se ainda a importância da manutenção de estações de coleta de dados, de modo a dar continuidade nos registros de precipitações diárias para que não haja falhas ou interrupção nas séries temporais, afetando a qualidade dos dados e estudos relacionados às precipitações.

Embora a tese das mudanças climáticas ainda ser polêmica, polarizando opiniões dos pesquisadores quanto à influência ou não das atividades humanas na alteração dos padrões climáticos globais, os estudos produzidos até então apontam que, além de aumento significativo da temperatura média global, haverá incremento de aproximadamente $20 \%$ das chuvas na região centro-sul do Brasil. Apesar desse futuro ainda incerto, esse incremento pode ser 
associado a um aumento de frequências de chuvas intensas em toda a região, além de chuvas intensas de maiores magnitudes, englobando também a cidade de Uberlândia-MG. Por fim, são sugestões que necessitam de mais estudos que revelem a previsibilidade das chuvas intensas, além de desenvolvimento de métodos estatísticos que levem em consideração o aumento nas temperaturas médias.

\section{AGRADECIMENTOS}

Agradecimento à Coordenação de Aperfeiçoamento de Pessoal de Nível Superior (CAPES), pelo apoio financeiro e suporte à pesquisa, visto que este trabalho é parte integrante de dissertação de mestrado.

\section{REFERÊNCIAS}

BACK, A. J.; OLIVEIRA, L. R.; HENN. A. Relações entre precipitações intensas de diferentes durações para desagregação da chuva diária em Santa Catarina. Revista Brasileira de Engenharia Agrícola e Ambiental, v. 16. n. 4, p. 391-398, 2012.

BERNARD, M. M. Formulas for rainfall intensities of long durations. Trans. ASCE, v. 96, 1932. p. 592- 624.

BERTONI, J. C.; TUCCI, C. E. M. Precipitação. In. TUCCI, C. E. M. (org). Hidrologia: ciência e aplicação. 3 ed. Porto Alegre: Editora da UFRGS/ABRH, 2004. p. 177- 242.

BRASIL, Ministério da Integração Nacional. Secretaria Nacional de Proteção e Defesa Civil. Centro Nacional de gerenciamento de Riscos e Desastres. Anuário brasileiro de desastres naturais 2013. Brasília: CENAD, 2014.

BRASIL, Ministério do Meio Ambiente, Ministério da Integração Nacional, WWFBrasil. Índice de vulnerabilidade aos desastres naturais relacionados às secas no contexto da mudança do clima. Brasília, DF: MMA, 2017. 125 p.

CAMPOS-ARANDA, D. F. Cálculo de las Curvas Intensidad-Duración-Período de Retorno, a partir de Registros de Lluvia Máxima en 24 horas y Relaciones Duración-Lluvia Promédio. Subdirección Regional Noreste de Obras Hidráulicas e Inginiería Agrícola para el Desarrollo Rural. San Luis Potosí, Septiembre 1978. $25 \mathrm{p}$.

CAVALCANTI, I. F. A. et. al. (orgs). Tempo e Clima no Brasil. São Paulo: Oficina de Textos, 2009.

CETESB - Companhia de tecnologia de saneamento ambiental. Drenagem urbana: manual de projeto. São Paulo: DAEE/CETESB, 1986. 466p.

HOLTZ, A. C. T. Precipitação. In: PINTO, N. L. S. et. al. Hidrologia Básica. São Paulo: Edgard Blücher, 1976. Cap. 2. p. 27-35.

INSTITUTO BRASILEIRO DE GEOGRAFIA E ESTATÍSTICA (IBGE) - Cidades. Disponível em: <https://cidades.ibge.gov.br/> acesso em: dez/2017

INSTITUTO NACIONAL DE PESQUISAS ESPACIAIS- INPE. Boletim do Grupo de Pesquisas em Mudanças Climáticas-GPMC- N. 8. Maio 2009. Edição Especial. 
Disponível

em:

<http://mudancasclimaticas.cptec.inpe.br/ rmclima/pdfs/newsletters/Boletim_ No8_Port.pdf>. Acesso em 01/11/2017.

KÖPPEN, W. Das geographisca System der Klimate. Gebr, Borntraeger, 1936, p. $1-44$.

KOUTSOYIANNIS, D.; KOZONIS, D.; MANETAS, A. A mathematical framework for studying rainfall intensity-duration-frequency relationships. Journal of Hidrology, v. 206, p. 118-135, 1998.

LAMBERT, D. M.; COOPER, M. C.; PAGH, J. D. Supply Chain Management: Implementation Issues and Research Opportunities. The International Journal of Logistics Management, v. 9, n. 2, p. 1-20, 1998.

LIEBMANN, B.; JONES, C.; CARVALHO, L. M. V. Interannual variability of daily extreme precipitation events in the State of São Paulo, Brazil. Journal of Climate, v. 14, p. 208-218, jan. 2001.

MACHADO, M. A. de M.; SILVA, E. D. B. Estimativa de chuvas intensas para o oeste de Minas Gerais e o entorno do reservatório da usina hidrelétrica de Furnas. Conexão Ciência (Online), v. 6, n. 2, p. 01-12, 2011.

MOHYMONT, B.; DEMAR'EE, G. R.; FAKA, D. N. Establishment of IDF-curves for precipitation in the tropical área of Central Africa - comparison of techniques and results. Natural Hazards and Earth System Science, v. 4, n. 3, pp.375-387, 2004.

MORUZZI, R. B.; OLIVEIRA, S. C. de. Relação entre intensidade, duração e frequência de chuvas em Rio Claro, SP: métodos e aplicação. Teoria e Prática na Engenharia Civil, v. 9, n. 13, p. 59-68, maio/2009.

NAGHETTINI, M.; PINTO, E. J. A. Hidrologia Estatística. Belo Horizonte: CPRM, 2007.

NERILO, N. Pluviometria e chuvas intensas no estado de Santa Catarina. 1999. Dissertação (Mestrado em Engenharia Ambiental) - Departamento de Engenharia Sanitária e Ambiental, Universidade Federal de Santa Catarina, Florianópolis, $174 \mathrm{f}$.

NOVAIS, G. T.; BRITO, J. L. S.; SANCHES, F. O. Unidades climáticas do Triângulo Mineiro/Alto Paranaíba. Revista Brasileira de Climatologia, ano 14, v. 23, Jul/Dez 2018, p. 223-243.

OLIVEIRA, L. F. C. de; et. al. Modelos de predição de chuvas intensas para o estado do Mato Grosso, Brasil. Revista Ambiente \& Água - An Interdisciplinary Journal of Applied Science: v. 6, n. 3, p. 274-290, 2011.

PETRUCCI, E. Características do clima de Uberlândia-MG: análise da temperatura, precipitação e umidade relativa. Dissertação. 2018. Dissertação (Mestrado em Geografia) - Instituto de Geografia, Universidade Federal de Uberlândia, Uberlândia, 2018.

PRUSKI, F.F.; SILVA, D.D.; TEIXEIRA, A.F.; CECÍLIO, R.A.; SILVA, J.M.A.; GRIEBELER, N.P. Hidros. Dimensionamento de sistemas hidroagrícolas. Viçosa: Editora UFV. 2006. 259 p. 
SANTOS, D. D. S.; GALVANI, E. Distribuição sazonal e horária das precipitações em Caraguatatuba-SP e a ocorrência de eventos extremos nos anos de 2007 a 2011. Ciência e Natura, v. 36 n. 2, Mai/Ago. 2014, p. 214-229.

SOUZA, R. O. R. de; et. al. Equações de chuvas intensas para o estado do Pará. Revista Brasileira de Engenharia agrícola e ambiental, v. 16, n. 9, p. 999-1005, 2012.

TANK, A. M. G. K.; ZWIERS, F. W.; ZHANG, X. Guidelines on analysis of extremes in a changing climate in support of informed decisions for adaptation. Climate Data and Monitoring WCDMP-No. 72. World Meteorological Organization, Geneva, Switzerland, Jun/2009, 56 p. Disponível em: https://library.wmo.int/doc_num.php?explnum_id=9419. Acesso em julho/2019.

TUCCI, C. E. M. (org). Hidrologia: ciência e aplicação. 3 ed. Porto Alegre: Editora da UFRGS/ABRH, 2004.

VAREJÃO-SILVA, M. A. Meteorologia e Climatologia. Versão Digital 2. Recife (PE), 2006. 449 p.

WEISS, L. L. Ratio of true to fixed-interval maximum rainfall. Journal of Hydraulics Division, v. 90, p.77-82, 1964.

WMO. World Meteorological Organization. Calculation of monthly and annual 30year standard normals. [Washington]: World Meteorological Organization, 1989. (World Climate Data Programme. WCDP n.10; World Meteorological Organization. WMO-TD n. 341). Disponível em: http://www.posmet.ufv.br/wpcontent/uploads/2016/09/MET-481-WMO-341.pdf. Acesso em agosto/2018. 